\title{
Space Internet Architectures and Technologies for NASA Enterprises 1
}

\author{
Kul Bhasin \\ NASA Glenn Research Center \\ 21000 Brookpark Road, MS 54-2 \\ Cleveland, Ohio 44135 \\ 216-433-3676 \\ kbhasin@grc.nasa.gov
}

\author{
Jeffrey L. Hayden \\ NASA Glenn Research Center \\ PresciPoint Solutions, L.L.C. \\ 5467 S. Cimarron Road \\ Littleton, CO 80123-2995 \\ 303-703-6911 \\ jlhayden@earthlink.net
}

\begin{abstract}
NASA's future communications services will be supplied through a space communications network that mirrors the terrestrial Internet in its capabilities and flexibility. The notional requirements for future data gathering and distribution by this Space Internet have been gathered from NASA's Earth Science Enterprise (ESE), the Human Exploration and Development in Space (HEDS), and the Space Science Enterprise (SSE).
\end{abstract}

This paper describes a communications infrastructure for the Space Internet, the architectures within the infrastructure, and the elements that make up the architectures. The architectures meet the requirements of the enterprises beyond 2010 with Internet compatible technologies and functionality. The elements of an architecture include the backbone, access, inter-spacecraft, and proximity communication parts. From the architectures, technologies have been identified which have the most impact and are critical for the implementation of the architectures.

\section{TABLE OF CONTENTS}

1. INTRODUCTION

2. Architectural Elements

3. Architectures For The Earth Science

ENTERPRISE (ESE)

4. Architectures For Human Exploration AND DeVelopment In Space (HEDS)

5. Architectures For The Space Science ENTERPRISE (SSE)

6. CONCLUSIONS

7. ACKNOWLEDGMENTS

8. REFERENCES

9. BIOGRAPHIES

\section{INTRODUCTION}

By analysis of missions in the conceptual phase and discussion with the NASA enterprises, the requirements beyond 2010 include high data rates, high capacity, interactivity with the in-space instrument, security of operations, real time data delivery, and seamless interoperability between in-space entities. The architecture that can be responsive to the requirement is the Open Standard Inter- connection (OSI) seven-layer model or its derivatives [1]. The advantages expected from this combination are significant increases in the quantity of data handled and the simplification of delivering that data to its destination. The improvements in data delivery are derived from the present capabilities and the continuous improvement in capability that commercial industry brings to the Internet without charge to NASA.

\section{Vertical Vs Horizontal Infrastructure}

NASA's communications infrastructure has grown out of the need to provide special services to each new mission as they were implemented. A vertically organized mission used infrastructure pieces that were designed to that single mission's requirements - resulting in communications assets only useful to that single mission. While it was possible to reuse some of the assets, such as the larger ground antennas, even these had to be modified to handle new mission requirements as they came along. Since this hardware was designed to operate at the lower frequencies (Sand X-band) and was not designed for flexibility, it has been increasingly difficult to bring the assets up to the capability of the commercial satellite systems that now operate at very high bit rates in the K-band.

NASA must consolidate its communications assets into a more horizontal infrastructure [2][3][4] so that the capabilities can be used to the advantage of any kind of mission that may wish to "plug-in" to it. The infrastructure must be revised to meet general specifications rather than meet any specific mission's requirements [5]. Missions would then be designed to interface to a general, highly capable, and standardized infrastructure. Once the infrastructure has been "flattened" and modified to provide high qualities of service to any mission type, further developmental activity will concentrate on continuous improvement of the capabilities of the infrastructure to the benefit of all missions.

NASA's technology programs have taken on the early development of the hardware needed to make the initial changes [6] to the infrastructure and enable it to leapfrog beyond the present commercial communications capabilities. However, NASA is also aware of and will take advantage of the capabilities offered by the Internet and its tech-

\footnotetext{
${ }^{1}$ U.S. Government work not protected by U.S. copyright
} 


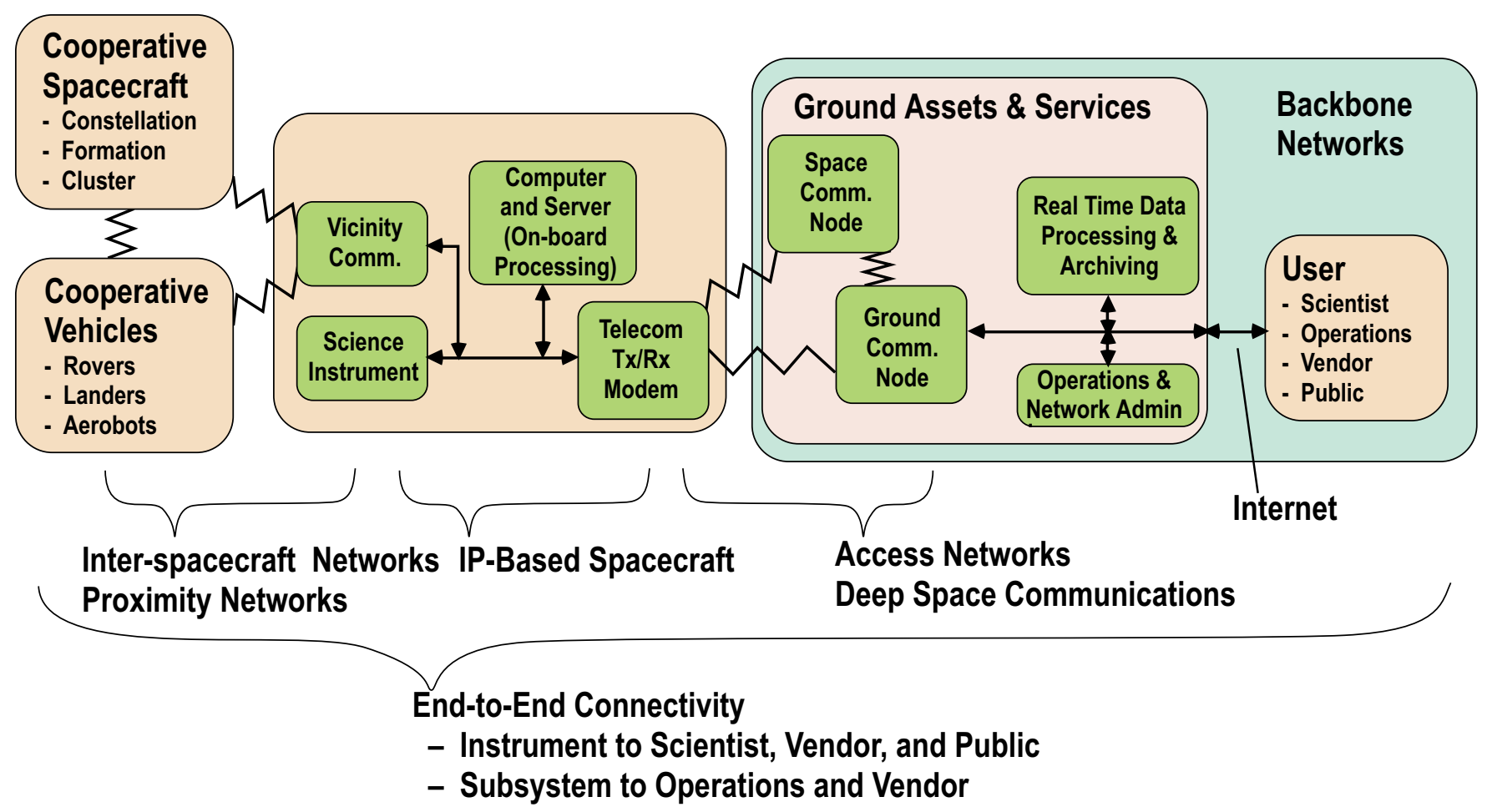

Figure 1, Simplified view of NASA's future communications infrastructure

nologies. One of the primary advantages of the Internet is that it is truly horizontal in its structure. That is, anyone can procure a computing device, connect to the Internet, and communicate through it because of the great flexibility and highly diverse set of open interoperability standards that the Internet conforms to and that are provided within each computing device.

This paper discusses the basic architectures identified and that are needed to support three of the NASA enterprises: the Earth Science Enterprise (ESE), the Human Exploration and Development in Space (HEDS), and the Space Science Enterprise (SSE). These architectures were inspected for similar aspects that were broken out as separate architectural elements.

Figure 1 displays a simple diagram of the extent of the infrastructure and the architectural elements, but without reference to the actual architectures. The figure shows the end-to-end connectivities between the scientists, the mission spacecraft, and instruments that NASA is striving for. Since this new infrastructure is based on Internet technologies, mission operations, hardware and spacecraft vendors, and the public (providing they are authorized), are afforded the same connectivity to NASA's exploration, science, and hardware.

The general infrastructure is discussed below and includes the following architectural element:

- Backbone network - the space network (SN), the ground network (GN), NASA's Intranets and virtual private networks (VPN's), the Internet, and any commercial or foreign communications system that may be employed:

- Access networks - the radio and/or optical communication interfaces between the backbones and the mission spacecraft and/or vehicles, and the local area networks (LAN's) on-board the spacecraft and/or vehicles;

- Inter-spacecraft networks - the radio and/or optical communication interfaces between spacecraft flying in a constellation, formation, or cluster;

- Proximity networks - the radio and/or optical communication interfaces between vehicles (rovers, airplanes, aerobots), landers, and sensors spread out in an ad hoc network.

\section{ARCHitectural ELEMENTS}

\section{Backbone Networks}

These networks, indicated in Figure 2, include NASA's GN and SN (the Tracking and Data Relay Satellite System - TDRSS) and any commercial satellite systems that might be employed to provide communications services to NASA spacecraft. The backbone network also includes the VPN's that tie NASA's assets together. Information available through the backbone network, includes data and tasking directives from: other spacecraft, vehicles, sensor networks, operations centers, archival databases, and users. The networks further enable pathways to foreign and domestic resources available on the Internet. Information access can significantly improve a mission's science by enabling coordination of activities and by providing data to a spacecraft to facilitate on-board processing. 


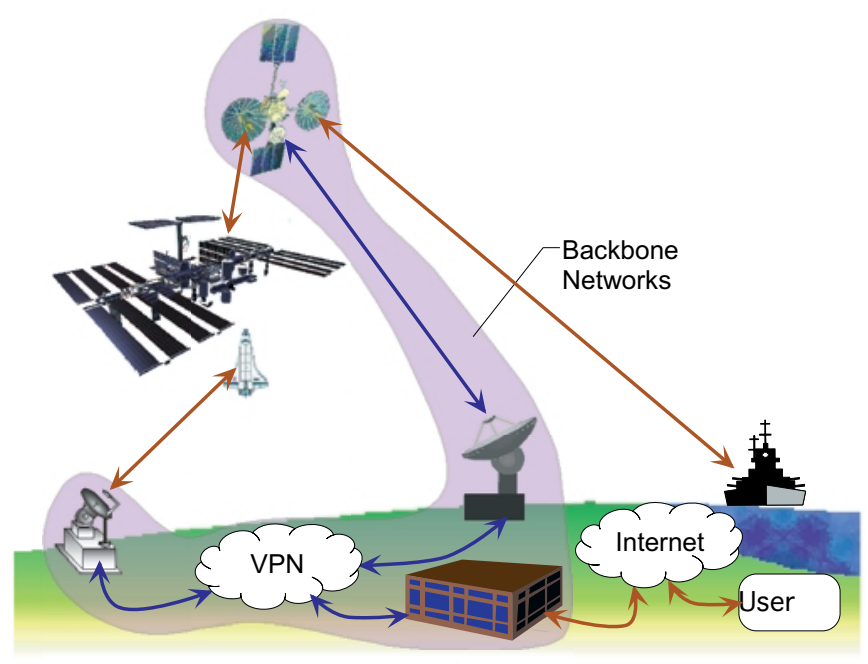

Figure 2, Backbone Network Elements

The data path through a backbone network is shown in Figure 3. The protocols levels that are used to handle the data that passes through the networks are shown on the left. The actual protocols to be used through the various network elements are under investigation. The unlabeled protocol levels are shown to give a sense of where protocol conversions are likely to occur in the networks.

Notionally, the user connects to the backbone via NASA's Intranet (by local Ethernet or dial-in connection), or through a secured route over the public Internet. The user and network switch shown on the left are at a NASA center on the NASA Intranet. The Intranet is connected to a ground station via a Virtual Private Network (VPN) allocated on a Wide Area Network (WAN) provided by a private company. Protocols that may be used on WAN's include Asynchronous Transfer Mode (ATM), Synchronous Optical NETwork (SONET), and frame relay. The ground station's Local Area Network (LAN) is most likely Ethernet or ATM. When the backbone is directly connected to a user spacecraft, the data can be directly passed on through to the user in real time, assuming the user is online or has an online autonomous system for capturing data. If the spacecraft is communicating with a NASA backbone network but the user is not connected to the backbone (e.g., when the WAN connection is down), tasking or science/telemetry data (depending on which entity is connected) is temporarily stored on a gateway server's fast Redundant Array of Inexpensive Disks (RAID) to be passed on later when a connection is established. User spacecraft connect to the backbone via modulated radio frequency or optical $(\mathrm{RF} / \mathrm{O})$ signals. The present interfaces are the antennas of the Ground Network (GN - NASA sites, foreign sites, and corporate sites), the Tracking and Data Relay Satellites (TDRS's) of NASA's Space Network (SN), and the antennas of the Deep Space Network (DSN).

\section{Access Networks}

These networks are the RF/O interfaces to the outer edges of the backbone networks for mission spacecraft, vehicles, and other entities. These interfaces, shown in Figure 4, include the remote entity's modem, receiver, transmitter, and antenna as well as the backbone"s matching set. Information can also be obtained by direct communication to other spacecraft or landed vehicle or stationary entities.

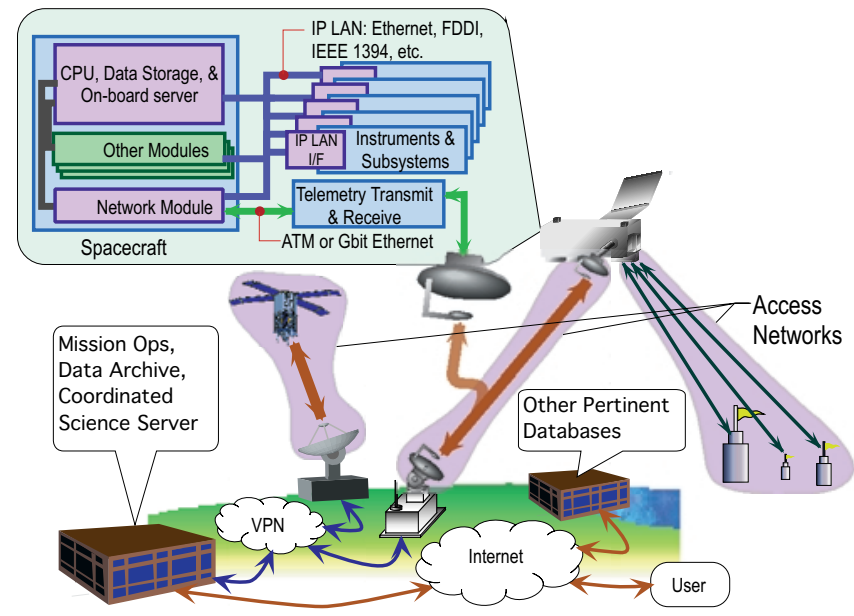

Figure 4, Access Network Elements

For instance, an over-flying earth observing spacecraft might access data from meteorological buoys and balloons and then integrate that data with its own observations of ocean waves, temperature, etc. to form complete data set files for download and use by anyone. Access networks provide the technologies for moving many of the data

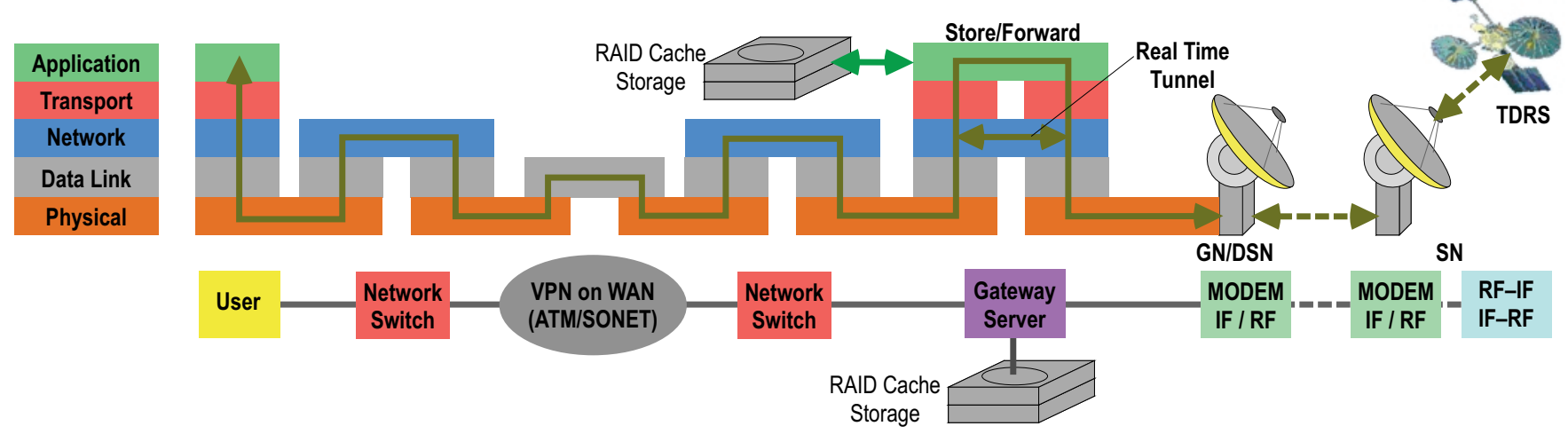

Figure 3, Backbone Network Model for GN, DSN, and SN 


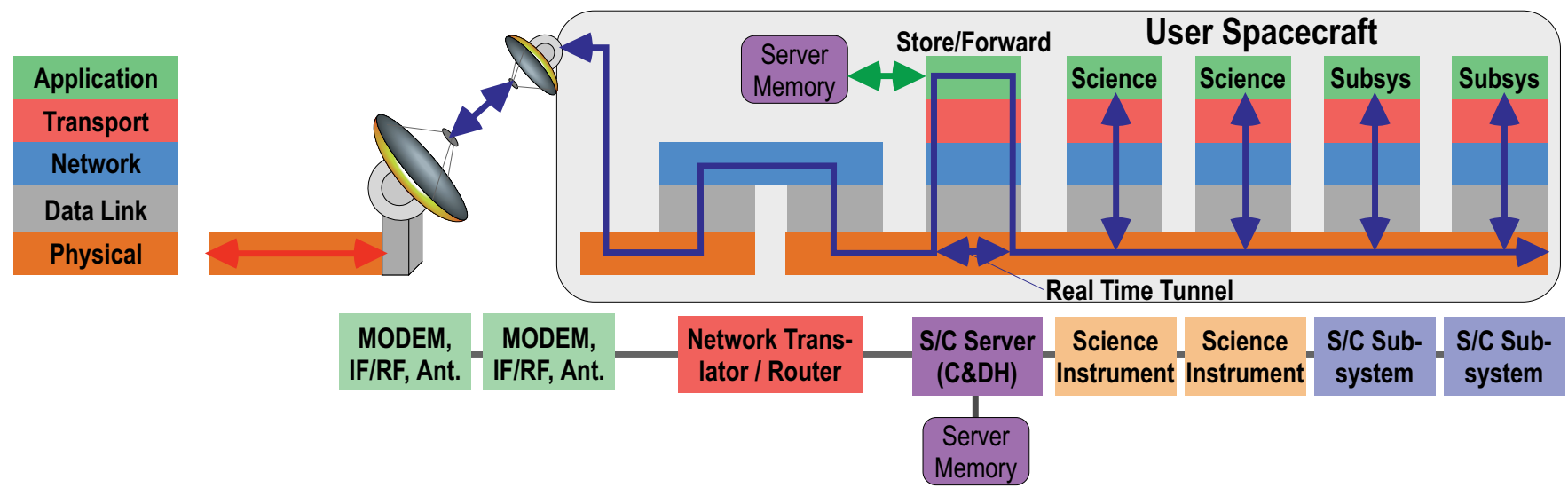

Figure 5, Access Network Model - for Spacecraft to Backbone

reduction tasks to the on-board processor.

As future on-board networks become compliant with Internet protocols (IP's) they will also come to resemble ground-based LAN's [3][4]. In the case of a science spacecraft, the on-board LAN (see Figure 5) is part of an access network that provides the interfaces for the science instrument to access information and services from the on-board resources. The information might include time and events data from the spacecraft subsystems, coordinated science data from other instruments, and overlay data provided from the ground or other spacecraft. Services provided by the spacecraft could then include science data file storage, communications, protocol translation, data routing, autonomous mission operations control, high level data product generation, and etc. Further, the instrument, via the onboard server and communication hardware, will access a backbone network node which connects the spacecraft to NASA's Intranet, the Internet, and ultimately the user. When the spacecraft is connected to the backbone, and the user is also online, real time communication with onboard LAN-connected instruments, and subsystems is possible. Communications that happen during times when the spacecraft is not connected to the backbone are handled by the on-board server. The server provides temporary storage for science data and autonomously passes that data to the backbone during connection periods.

\section{Inter-spacecraft Networks}

Spacecraft that fly in cooperation, such as in constellations, tight formations, or loose clusters, incorporate an inter-spacecraft network for local communications and coordination. Such geometries may use a wireless interface (radio or optical) or a tether interface (wire or fiber optic) to interconnect neighboring spacecraft. In many concepts the inter-spacecraft network also takes part in the measurement of relative position between spacecraft.

A few geometries for formation and cluster flying are shown in Figure 6. It is possible to assign several backbone ground stations to communicate with each spacecraft in a formation or cluster simultaneously. This type of operation is difficult to coordinate, however, and stretches the capability of the backbone. For that reason, a good solution is to assign a single member of the formation to handle the access network interface responsibilities between the formation and the backbone network. Tight flying star formations that use a single spacecraft to coordinate the operation and control of the formation's mem-

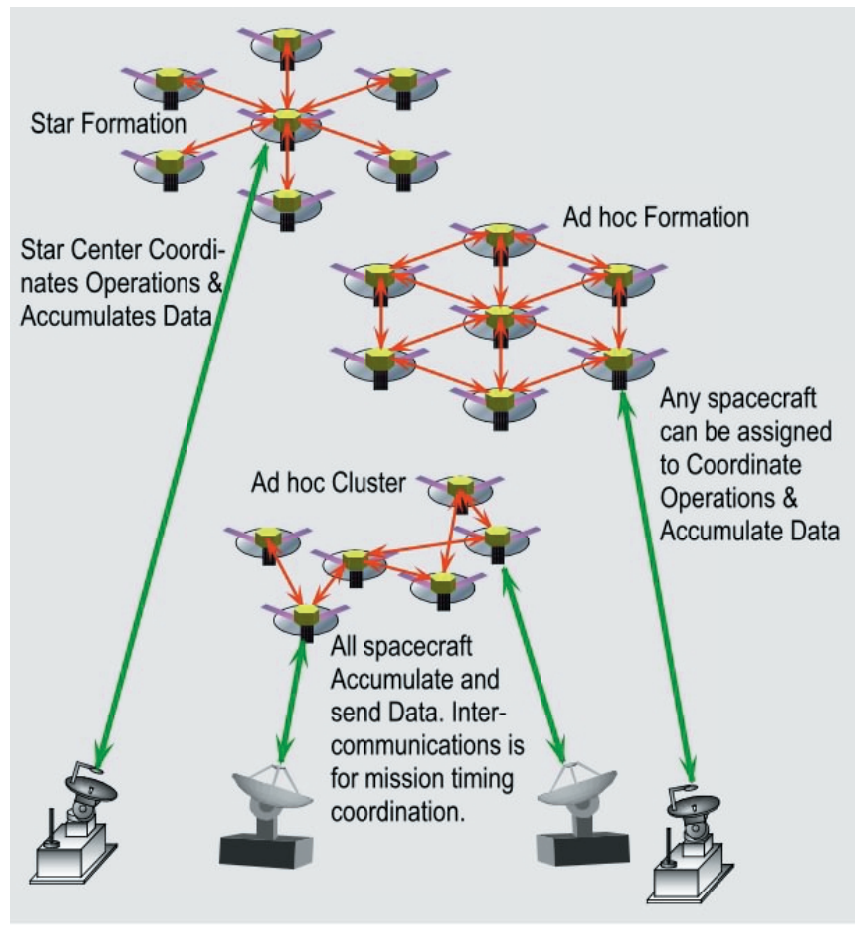

Figure 6, Inter-spacecraft Network Elements

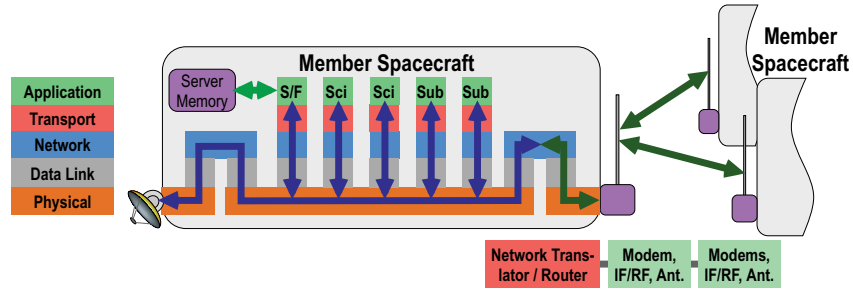

Figure 7, Inter-Spacecraft Network Model for Spacecraft to Spacecraft 
bers might also use that spacecraft to handle the communications tasks.

Other solutions might pass the communications interface off to any one member at a time, if that member is part of an ad hoc formation or cluster. In the ad hoc case, the member that connects to the backbone could act as a "bent pipe" connection for the other members to send their data to the backbone. The figure shows only a few possible coordinated structures. NASA will continue investigating these and other structures to find optimum ways of operating each, identifying the technologies needed to make them work, and synthesizing requirements for those technologies.

If the inter-spacecraft links are implemented as attachments to each spacecraft's LAN, as shown in Figure 7, then direct communications between instruments and subsystems on different spacecraft are facilitated. This scheme seems quite flexible and might be used to pass data between spacecraft so as to distribute the overall data processing load.

\section{Proximity Networks}

Closely spaced, landed and airborne entities (vehicles, stations, sensors, balloons, etc.) will intercommunicate using low power proximity networks in an ad hoc fashion.

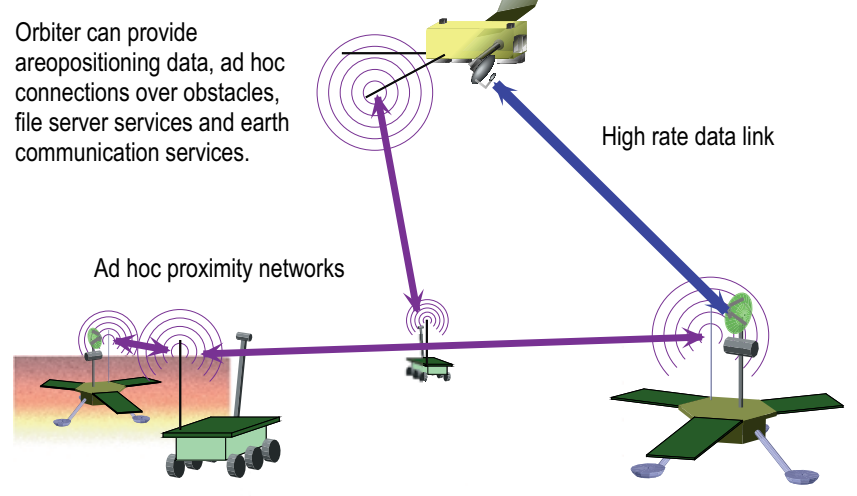

Figure 8, Proximity Network Elements
As shown in Figure 8, entities in a local area form a flexible network to pass data between them and to pass data to entities designated as coordinating nodes. Over-flying orbiters can act as short term members of the landed ad hoc group by passing data between entities that cannot "see" each other well enough to communicate. The overflyers would also relay the group's finished data to earth and pass mission tasks from earth to the group.

The proximity network protocol structure shown in Figure 9 is very similar to the inter-spacecraft structure shown in Figure 7. The data rates will likely be somewhat lower due to the use of low power consumption communication and computing components.

At higher data rates this ad hoc proximity network concept also provides a model for handling communications in the vicinity of the International Space Station (ISS). As activities around ISS increase with Shuttle and Soyuz arrivals/departures and human/robotic maintenance and build activities, the local communications system must be able to autonomously reconfigure to accept new arrivals and drop-off those that leave. This proximity network can then be used to provide communications between all moving entities as well as provide a means of warning of impending collisions. The same network would provide video connectivity for an ISS coordinator to monitor/guide robot or human activity.

\section{Architectures For The EArth Science ENTERPRISE (ESE)}

The technologies for the Earth Science Enterprise (ESE) missions enable very high rate data transfers between the ground and low earth orbit (LEO), the Moon, and the vicinity of Earth's orbit around the sun. The new technology initiatives [1] in high bandwidth microwave and optical links and in the space Internet enable Direct Data Distribution (D3) to the scientist and public, High Rate Imagery Processing and Delivery (HRIPD), and the gathering of specialized products for environmental monitoring and forecasting. Lightning/rain correlations, storm, fire, and plume/gas tracking, crop and ocean health and pollution monitoring, and etc. are all improved with near real time

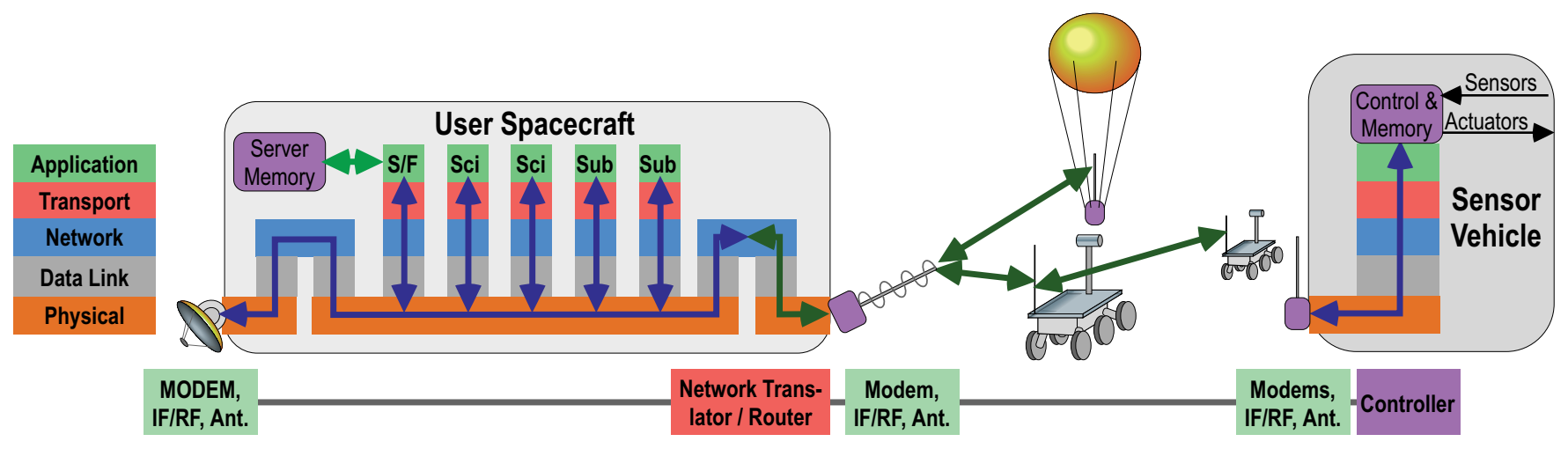

Figure 9, Proximity Network Model - for Sensor Net (Landers/Stations, Rovers, Aerobots, Airplanes, etc.) and to Relay Spacecraft 
finished data product availability to any interested person on the Internet.

Figure 10 shows the general architecture of the ESE communications networks. Missions use the access and space and/or ground backbone networks to pass high rate data, low rate telemetry, and commands between the spacecraft and ground. Missions supported by ESE networks include the Earth Observing System (EOS), Triana, Geospace Electrodynamics Connections (GEC), NOAA weather satellites, Landsats, atmospheric chemistry and physics experiments, earth plasma and solar wind interaction experiments, and others.

The figure indicates a future in which a mission might use, at its convenience, the $\mathrm{SN}$, or a commercial communications satellite system, or the GN. NASA is developing the standard interface technologies for the access and backbone networks that are needed to enable a spacecraft to connect through any network system. Whether or not a mission needs the flexibility to connect to any backbone is then left up to the mission development team. The plan is to have the capability in-place.

The technologies for inter-spacecraft networks will apply to any cooperative multi-spacecraft missions that are implemented. Missions of this type being considered include: Magnetospheric Constellation (MC), Magnetospheric Multiscale (MMS), and the Nanosat Constellation Trailblazer (ST5).

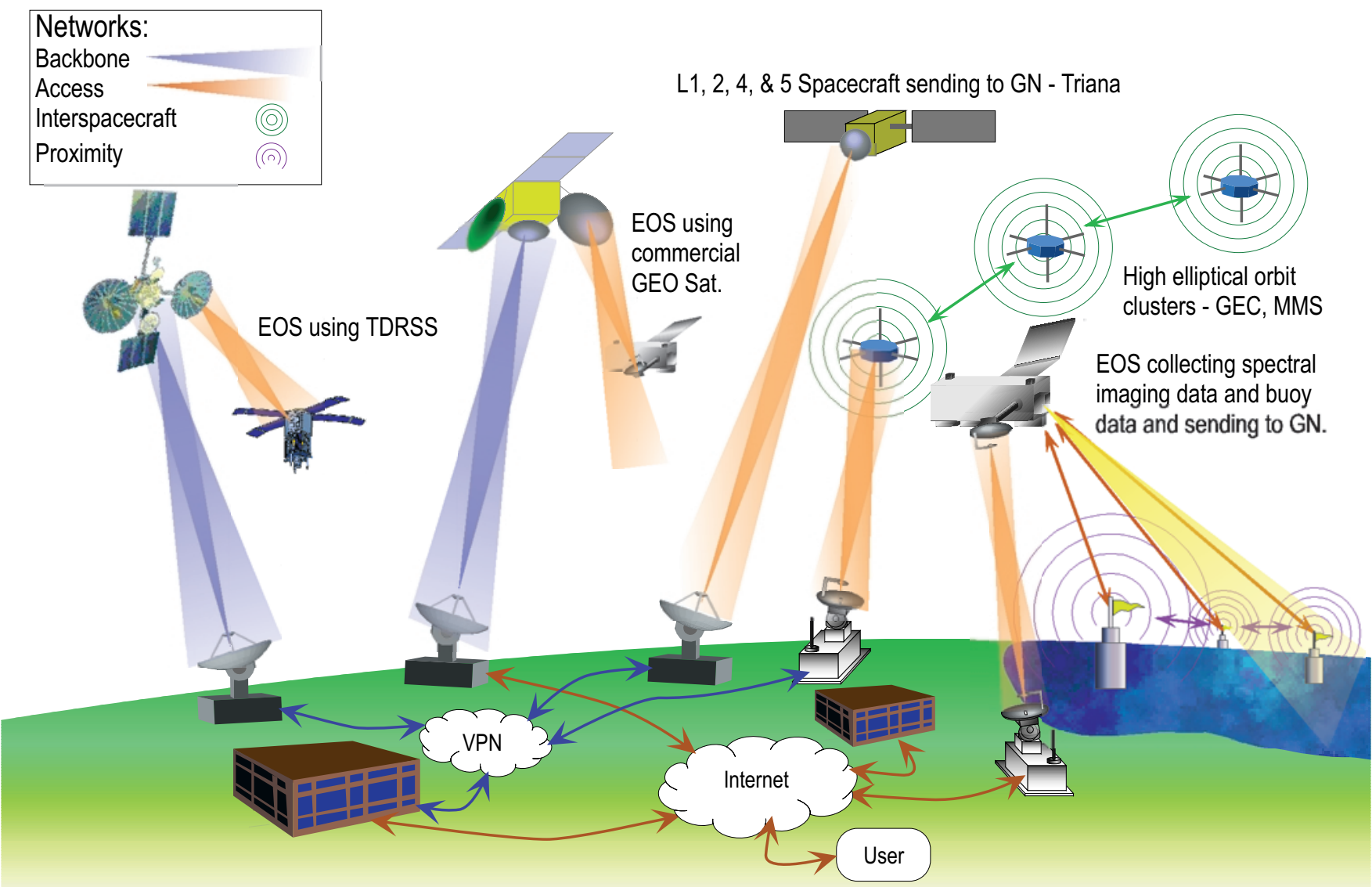

ESE will also benefit from work on low power proximity networks. Ground, sea, air, and space sensors that measure Earth's parameters will communicate with each other and with data gathering spacecraft using these technologies.

\section{Architectures For Human ExPloration AND DEVELOPMENT IN SPACE (HEDS)}

Architectures for the HEDS enterprise (Figure 11) will likewise benefit from the very high data rate transfer capabilities of the future networks. These technologies provide clear examples of the benefits of developing for the general benefit of the NASA enterprises rather than for specific mission requirements - all missions get a better overall system. General technologies that benefit HEDS include $\mathrm{Ka} / \mathrm{Q} / \mathrm{V}$-band and optical technologies, utilization of commercial satellites, and improved utilization of government communication satellites. Other features of general utility are D3 to the scientist and public, and IP-based communications for shuttle/ISS which will handle voice, video, data, and emerging commercial and military broadband services.

NASA is also investigating a Very High Rate Data (VHRD) augmentation to the backbone networks that will ensure near continuous connection of ISS to the Internet at several Gbps. This augmentation comprises a spacecraft that flies in the orbit of but trails ISS at a safe $1000 \mathrm{~km}$ or so. The spacecraft communicates with ISS with a modulated optical beam. It then serves as a router by sending (in

Figure 10, ESE Architectures 


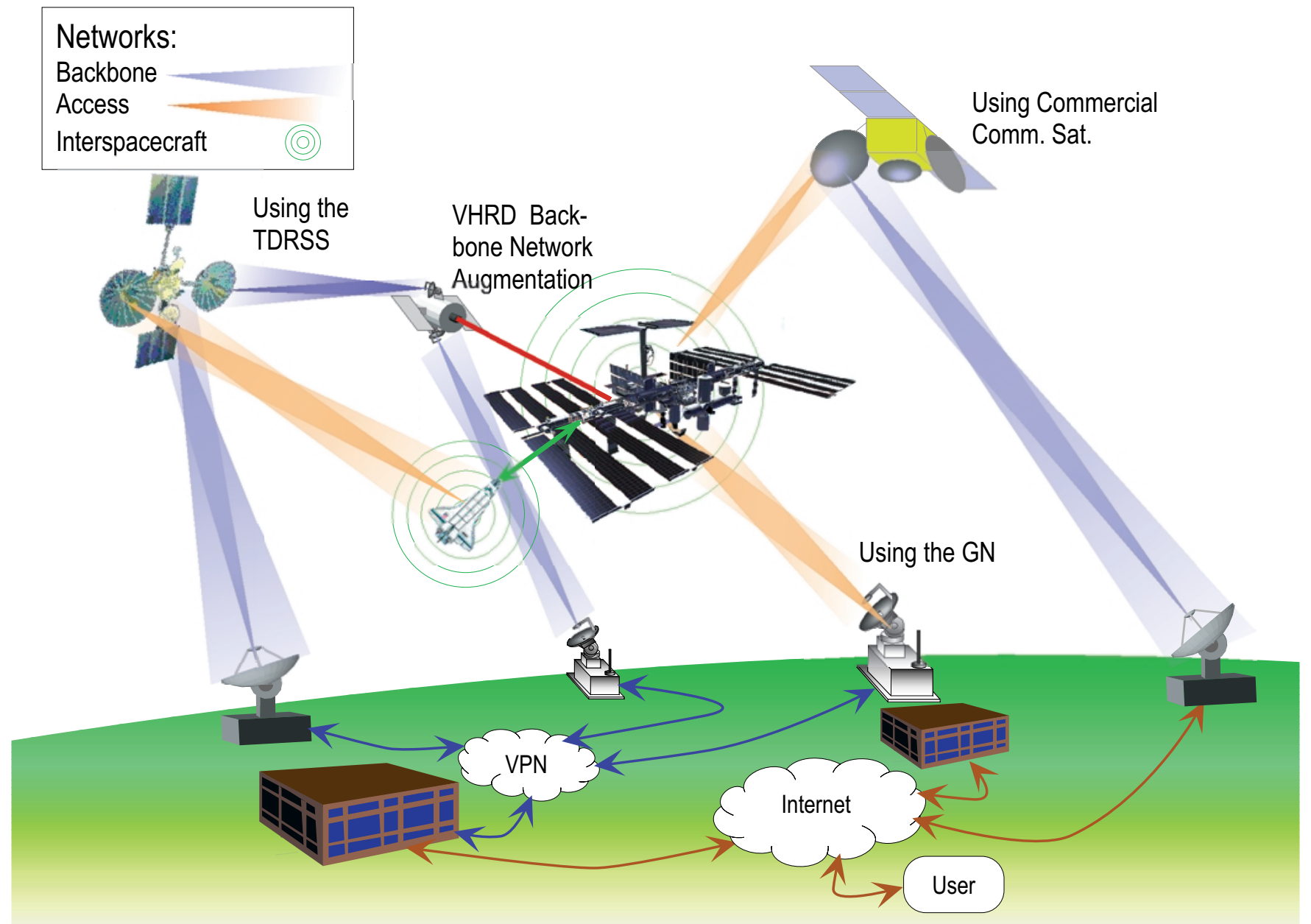

Figure 11, HEDS Architectures

the Ka-band) data up to an available TDRS or commercial communications satellite or down to a ground station.

Self forming and dissolving ad hoc proximity networks may be necessary to the coordination and safety of work in the external ISS vicinity. These ad hoc networks are an important part of several mission concepts under study in the other enterprises. It's likely that common solutions can be found that would be inherently superior to re-inventing these networks for individual missions. Solutions already emerging from the commercial sector are the IEEE 802.11 and the European originated Bluetooth specifications as well as the open and the proprietary specifications for the cell phone environment. IEEE 802.11 and Bluetooth are tailored for communication between multiple computers in a slow mobile environment. Bluetooth generally operates below $1 \mathrm{Mbps}$ but IEEE 802.11 can operate at respectable speeds ranging from 2 to $11 \mathrm{Mbps}$ with future implementations going above $50 \mathrm{Mbps}$. At these speeds, members in an ad hoc group around the vicinity of the ISS can easily pass voice and video between them to enhance safety and improve in-space work effectiveness. These specifications handle communications in an ad hoc group by autonomously picking up new members and dropping off those that pass out of the range of the group.

\section{Architectures For The Space Science ENTERPRISE (SSE)}

Technologies for the SSE are derivations from the technologies being developed for the other enterprises [1][4]. Deep space missions and missions to Mars will also take advantage of the high bandwidth microwave and optical links being developed for general use. The Mars Network will eventually employ surface-to-orbiter access links and surface-to-surface proximity networks, as well as the Kband and optical link technologies that are also being developed for ESE and HEDS.

The Earth-side networks for SSE are shown in Figure 12. These include the GN that is used to communicate with the in-space observatories for the Origins and Sun - Earth Connection programs and the Deep Space Network (DSN) that is used to communicate with spacecraft normally beyond $2 \mathrm{AU}$. The in-space observatories are placed in the vicinity of Earth - from LEO out to the libration points at L1, L2, L4 and L5. The access networks for these observatories will utilize Ka-band communications and become IP compliant such that data from the observatories will be available to scientists and the public in near real time. The networks will enable each using scientist, in turn, to have full control of an observatory via the Internet. 


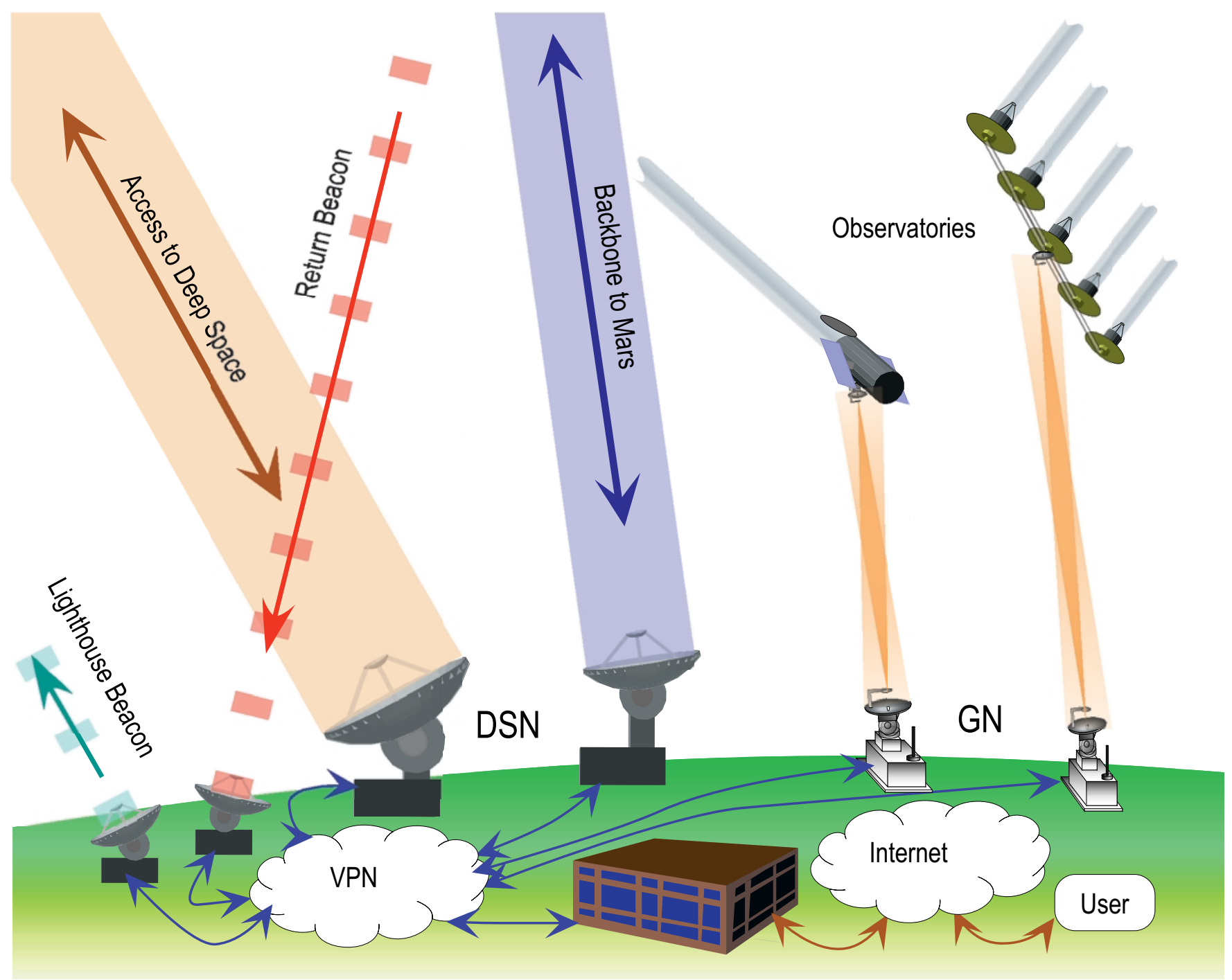

Figure 12, Earth-side Networks for the SSE.

Ground stations located at Goldstone, California, Canberra, Australia, and Madrid, Spain comprise the Earth-side of the DSN. The larger antennas, $34 \mathrm{~m}$ and $70 \mathrm{~m}$, form part of the backbone network to Mars and the access network for other deep space mission spacecraft. The DSN is being and will continue to be upgraded to Ka-band operation. Optical communications from telescopes atop mountains may also be added as a DSN capability.

Communications delay times to deep space missions become too long for real time human interaction with a mission during critical and emergency operations. Because of this, mission spacecraft must perform critical and emergency procedures autonomously. A beacon mode system will be implemented at the DSN sites to assist these autonomous operations and to reduce the overall cost of operations. The beacon system is implemented with smaller diameter antennas that operate at very low bit rates. Some antennas are operated as lighthouse beacons that send timing and ranging return signals to spacecraft. A spacecraft receiving this beacon signal can synchronize its clock with earth-time and can determine its distance from earth by timing the return of a signal it has sent to a receiving beacon and that was sent back by the lighthouse beacon. The spacecraft can also use one-way Doppler to determine its velocity relative to earth.

A spacecraft will also send a beacon signal back to earth to indicate the state of its health and to request communications service from the DSN. This part of the beacon system will save costs by utilizing smaller antenna assets for the task of monitoring many spacecraft during the quieter phases of their missions and by autonomously calling in the larger assets only when needed for emergency or for support of mission critical data reception.

A backbone network between Earth and Mars is shown in Figure 13. The present thoughts for this network are that three communications spacecraft will be put in high altitude, high inclination orbits (but not in Polar orbit) about Mars. These spacecraft will usually be able to see each other and will cover fixed places on the surface for 


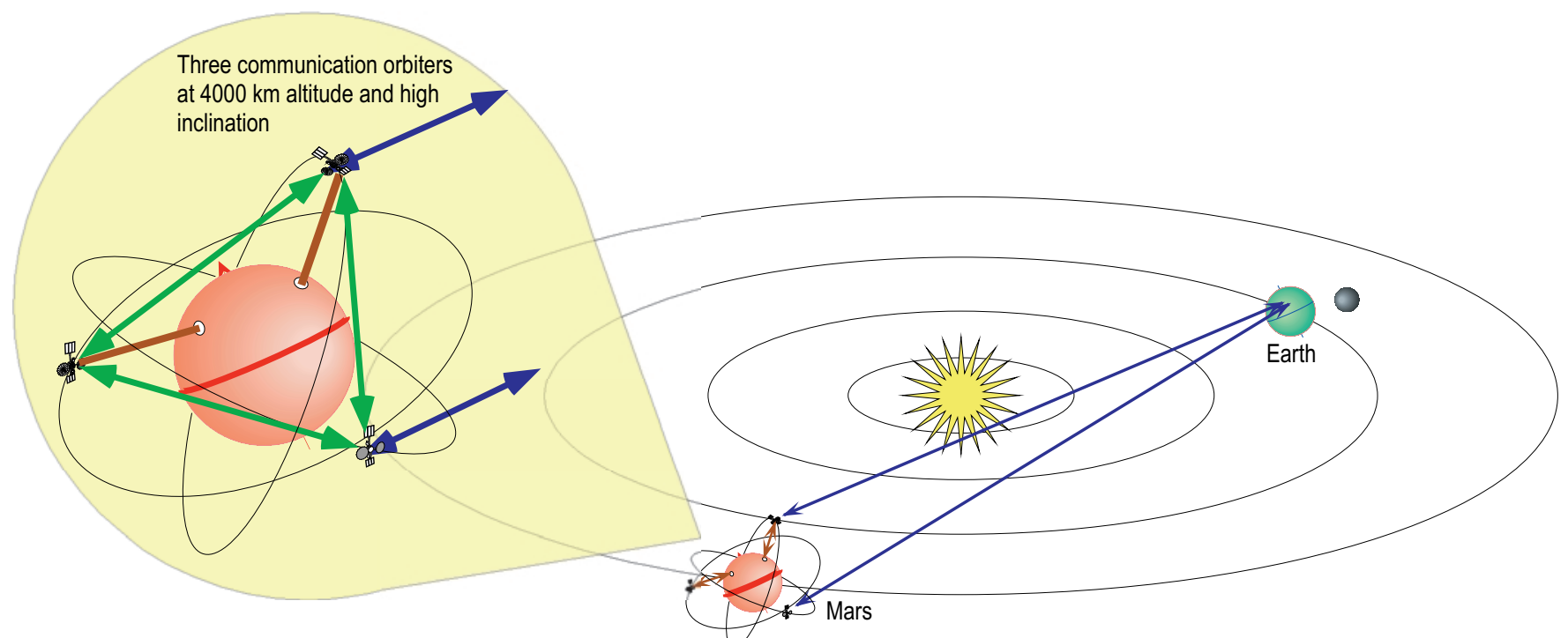

Figure 13, A Mars Communication Architecture

reasonably long periods of time. Most places on the surface will be in view of an orbiter most of the time. This simple constellation can support many surface and balloon activities at a time. These spacecraft will incorporate storeand-forward servers to temporarily hold data for transfer to another surface element or to the Earth. The spacecraft may also include inter-spacecraft communications links to pass data around Mars from one surface site to another or to the Earth.

In the more distant future, the solar system communication network shown in Figure 14 could be implemented. To implement this system, it is assumed that most of the large flexible structure technologies are in place so that large but lightweight communications systems can be placed in space at reasonable cost. In this concept, large antenna structures are tethered together with a control spacecraft and a large solar array. The solar array powers all the units through wires in the tethers. The control unit acts as a router for sending data received from earth through the appropriate antenna to a mission's spacecraft and for handling the mission's data return route. All units handle their own

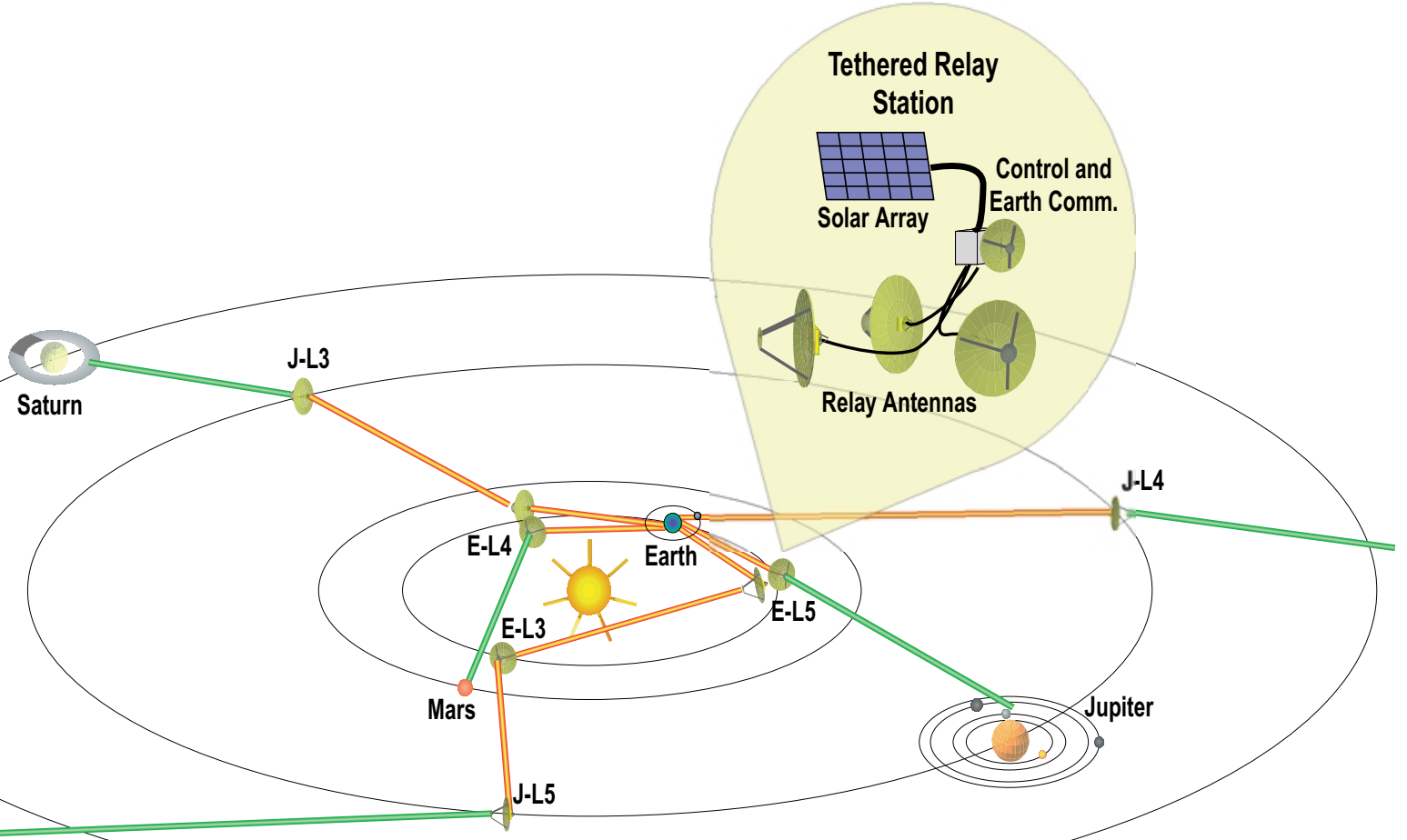

Figure 14, An Architecture for High Data Rate Communications to Deep Space 
pointing and the central control unit ensures that the units don't collide.

These tethered relay stations can be placed at various Lagrangian libration points such as the Earth and Jupiter L3, L4 and L5 points. Such relay stations could vastly improve the communications data rates to distant locations in the solar system.

\section{CONCLUSIONS}

This paper has described a set of four architectural elements that can be assembled into end-to-end communications architectures to satisfy the real and perceived requirements for future missions of NASA's enterprises: ESE, HEDS, and SSE. The suggested elements are the backbone, access, inter-spacecraft and proximity networks. By deriving each element's functionality from the Earth-bound Internet's technologies and protocols, cost savings can accrue from common use of technologies; and the advantages of D3 can be realized without the need for human assistance along the route.

It is intended that these elements might serve as a basis for discussion of future architectures and for synthesizing the requirements of those architectures. Rather than develop entire new infrastructure to support a single mission, it is desirable to define and implement new architectural elements that can be evolved over time by adding new capability to service the new mission as well as any other new mission that may need similar services. The elements can then be utilized in a uniform way across the enterprises with variations only in those areas that are specific to a single enterprise ( e.g.; the very high data rates to handle multiple streams of ISS video, voice, and scientific activity; and the communication technologies needed to accommodate the very long delay times and weak signals associated with deep space missions).

Some architectural constructs have been posed for future consideration. Two of these are the high-speed proximity network in the vicinity of the ISS and the solar system communications network that is enabled with inflatable structures and tethered clusters.

The implementation of advanced architectures will require high capacity, high rate, reliable microwave or optical communication technologies for the backbone for near Earth and deep space. Primary enablers for these advanced architectures include miniaturization of reliable network technologies and mobile protocols that are delay insensitive and operate with minimum data overhead. All in-space architectural elements will, in general, require highly miniaturized, low power, mass and volume network and wireless hardware.

\section{ACKNOWLEDGMENTS}

The authors would like to thank Grant Runyon and Mary Fletcher for their assistance.

\section{REFERENCES}

[1] Kul Bhasin, Ramon P. De Paula , Charles D. Edwards, Internet technologies for Space-Based Communications: state of the Art and Challenges, 18th AIAA International Communications Satellite Systems Conference, Oakland, CA, April 10-14, 2000

[2] "Section 4, Space/Ground Trades", Consolidated Space Operations Contract Architecture Baseline, CSOC document, December 1998.

[3] Jeff Hayden, "Telecommunications for the CSOC, an Internet Interface to NASA Satellites", 1999 Second Annual International Symposium On Advanced Radio Technologies, September 8-10, 1999

[4] Jeffrey L. Hayden, Spacecraft/Ground Architectures using Internet Protocols to Facilitate Autonomous Mission Operations, 2000 IEEE Aerospace Conference, March 2000.

[5] "Appendix C, Candidates For Spacecraft / Ground Tradeoffs By Functional Area", Consolidated Space Operations Contract Architecture Baseline, CSOC document, December 1998.

[6] High Rate Data Delivery website: http://hrdd.grc.nasa.gov

\section{BIOGRAPHIES}

Dr. Kul B. Bhasin serves as the Space Technology Manager for High Rate Data Delivery for the Space Base Technology Program in the Aero-Space Technology Enterprise. In this position he is responsible for the development and integration of advanced space communication and network technologies to meet future needs of NASA enterprises.

Prior to that he established and was Chief of the Satellite Networks and Architectures Branch at NASA Glenn Research Center, Cleveland, OH. He initiated several joint-working groups with industry and academia to develop space Internet working standards. He served on the NSF/NASA blue ribbon panel to assess the Global Satellite Communications Technology and Systems. He represented NASA in the Ad Hoc Satellite Industry Task Force to define the role of satellites in the Global Information Infrastructure.

Dr. Bhasin is a senior member of IEEE and is an elected Fellow of the Society of International Optical Engineers (SPIE). He is a member of AIAA and also serves on the Satellite Communication Technology Committee for AIAA. He was a NASA Fellow at Cornell University in Electrical Engineering in 1985. He obtained his PhD and MS 
degrees from the University of Missouri and Purdue University, respectively.

Jeff Hayden retired as an aerospace Lockheed Martin Astronautics in Denver. He had worked on NASA's network design for the CSOC program's Integrated Operations Architecture focusing on implementing Internet Protocols at the science instrument and on-board the spacecraft. He has described new spacecraft architectures that would oper-

ate in an Internet environment. Mr. Hayden has also performed initial spacecraft system designs for Stardust, Genesis, and Space Based Laser. Jeff's original expertise was as an instrument designer. In the sixties, he designed miniature mass spectrometers flown on Aerobee sounding rockets and on the Atmosphere Explorer $C$, D, and E satellites for the University of Minnesota. He designed the prototype for the Upper Atmosphere Mass Spectrometer for the Viking missions in the early '70's. While at Lockheed Martin, he performed the instrument system designs for the Net Flux Radiometer for Galileo Probe and for the Gamma Ray Spectrometer for Mars Observer. 